\title{
Novel Aspects of Tomato Root Colonization and Infection by Fusarium oxysporum f. sp. radicis-lycopersici Revealed by Confocal Laser Scanning Microscopic Analysis Using the Green Fluorescent Protein as a Marker
}

\author{
Anastasia L. Lagopodi, ${ }^{1}$ Arthur F. J. Ram, ${ }^{1}$ Gerda E. M. Lamers, ${ }^{1}$ Peter J. Punt, ${ }^{2}$ \\ Cees A. M. J. J. Van den Hondel, ${ }^{1,2}$ Ben J. J Lugtenberg, ${ }^{1}$ and Guido V. Bloemberg ${ }^{1}$ \\ ${ }^{1}$ Leiden University, Institute of Molecular Plant Sciences, Wassenaarseweg 64, 2333 AL, Leiden, The Netherlands; \\ ${ }^{2}$ TNO Nutrition and Food Research Institute, Utrechtseweg 48, 3500 AJ Zeist, The Netherlands
}

Submitted 16 May 2001. Accepted 12 October 2001.

The fungus Fusarium oxysporum f. sp. radicis-lycopersici is the causal agent of tomato foot and root rot disease. The green fluorescent protein (GFP) was used to mark this fungus in order to visualize and analyze the colonization and infection processes in vivo. Transformation of $F$. oxysporum f. sp. radicis-lycopersici was very efficient and $g f p$ expression was stable for at least nine subcultures. Microscopic analysis of the transformants revealed homogeneity of the fluorescent signal, which was clearly visible in the hyphae as well as in the chlamydospores and conidia. To our knowledge, this is the first report in which this is shown. The transformation did not affect the pathogenicity. Using confocal laser scanning microscopy, colonization, infection, and disease development on tomato roots were visualized in detail and several new aspects of these processes were observed, such as (i) the complete colonization pattern of the tomato root system; (ii) the very first steps of contact between the fungus and the host, which takes place at the root hair zone by mingling and by the attachment of hyphae to the root hairs; (iii) the preferential colonization sites on the root surface, which are the grooves along the junctions of the epidermal cells; and (iv) the absence of specific infection sites, such as sites of emergence of secondary roots, root tips, or wounded tissue, and the absence of specific infection structures, such as appressoria. The results of this work prove that the use of GFP as a marker for $\boldsymbol{F}$. oxysporum f. sp. radicis-lycopersici is a convenient, fast, and effective approach for studying plant-fungus interactions.

Additional keywords: autofluorescent protein, biocontrol, rhizosphere, Pseudomonas, tomato crown and root rot.

Corresponding author: G. V. Bloemberg; Telephone: +31-71-5275056; Fax: +31-71-5275088; E-mail: bloemberg @ rulbim.leidenuniv.nl

Current address of A. L. Lagopodi: Aristotelian University of Thessaloniki, Faculty of Agriculture, Plant Pathology Laboratory, P.O. Box 269, 54006 Thessaloniki, Greece; E-mail: lagopodi@agro.auth.gr

* The $\boldsymbol{e}$-Xtra logo stands for "electronic extra" and indicates the HTML abstract available on-line contains supplemental material not included in the print edition. There is a video clip available on-line.
Fusarium oxysporum f. sp. radicis-lycopersici is the causal agent of tomato foot and root rot (synonym for crown and root rot), a disease of worldwide economic importance in commercial tomato production (Brayford 1996; Jarvis 1988). The disease results in severe losses in the greenhouse, open field crops, and hydroponic cultures. Like all formae speciales of $F$. oxysporum, this fungus is a soil inhabitant and extremely difficult to control. More detailed knowledge on the in vivo interactions between the pathogenic fungus and the plant could lead to the discovery of more efficient ways to control the disease. Details of these interactions can be essential in studies of the biological control of the fungus by beneficial antagonistic microorganisms that colonize the tomato rhizosphere as well (ChinA-Woeng et al. 1997, 1988; Dekkers et al. 2000; Lugtenberg et al. 2001).

The green fluorescent protein (GFP) from the jellyfish Aequorea victoria (Chalfie and Kain 1998; Tsien 1998) was used to label $F$. oxysporum f. sp. radicis-lycopersici in order to visualize by confocal laser scanning microscopy (CLSM) its behavior in the tomato rhizosphere under in vivo conditions. GFP's fluorescence is stable and species independent and does not require any cofactors or substrates. Visualization of GFPlabeled organisms with CLSM is an effective, fast, and noninvasive tool that allows the spatiotemporal analysis of interactions while preserving the integrity of the organisms under study. In this paper, we describe (i) the transformation of $F$. oxysporum f. sp. radicis-lycopersici with a plasmid-harboring $g f p$ that results in stable expression of $g f p$, (ii) the demonstration that GFP is an excellent reporter in $F$. oxysporum f. sp. radicislycopersici for visualizing this fungus in the tomato rhizosphere, and (iii) novel aspects of the colonization and infection processes on tomato roots by $F$. oxysporum f. sp. radicis-lycopersici.

In order to express $g f p$ in $F$. oxysporum f. sp. radicislycopersici, the sgfp gene (Chiu et al. 1996) was cloned between the Aspergillus nidulans gpdA promoter (Punt et al. 1988) and the trpC terminator (Mullaney et al. 1985) sequences as follows. Plasmid pAN52-10-S65TGFPn/n (Siedenberg et al. 1999), which contains the sgfp gene between the glaA promoter and the $\operatorname{trpC}$ terminator, was digested with $N c o$ I and Not $\mathrm{I}$ in order to remove the glaA promoter. The gpdA promoter was isolated from pAN52-1Not (Van den Hondel et al. 1991) as a $2.3-\mathrm{kb} N \operatorname{coI}-N o t \mathrm{I}$ fragment and ligated into NcoI-NotIdigested $\mathrm{pAN} 52-10-\mathrm{S} 65 \mathrm{TGFPn} / \mathrm{n}$ to give $\mathrm{pGPDGFP}$. The con- 
struction of plasmid pGPDGFP to express gfp in F. oxysporum f. sp. radicis-lycopersici was carried out using standard cloning techniques (Maniatis et al. 1982) and is schematically represented in Figure 1. pAN7-1 (Punt et al. 1987) carrying the Escherichia coli hygromycin-B (Hm-B) resistance gene hph, cloned also between the Pgpd promoter and $\operatorname{trp} C$ terminator, together with pGPDGFP, were used in a cotransformation of $F$. oxysporum f. sp. radicis-lycopersici. pAN7-1, which encodes for $\mathrm{Hm}-\mathrm{B}$ resistance, allows selection of transformants on media containing this antibiotic.

The virulent isolate of $F$. oxysporum $\mathrm{f}$. sp. radicis-lycopersici, CBS 101587 (deposited in the Centraal Bureau voor Schimmelcultures, Baarn, The Netherlands), was transformed and used for artificial inoculations on tomato. The fungus was maintained at $-80^{\circ} \mathrm{C}$ in $10 \%$ glycerol and was routinely grown at $22^{\circ} \mathrm{C}$ on potato dextrose agar (PDA) (Difco Laboratories, Detroit, MI, U.S.A.) supplemented with $0.1 \mathrm{mg}$ of streptomycin per $\mathrm{ml}$ and $0.01 \mathrm{mg}$ of tetracycline per $\mathrm{ml}$ to eliminate bacterial contaminations. The fungus was transformed by a polyethyleneglycol/ $\mathrm{CaCl}_{2}$-mediated transformation of protoplasts as described by Kistler and Benny (1988) and modified by Mes and colleagues (1999) with the following additional modifications. (i) Digestion of mycelial cell walls was achieved by Lysing Enzymes (10 mg/ml, Sigma L-2265; Sigma Chemical Co., St. Louis, MO, U.S.A.) in $1.2 \mathrm{M} \mathrm{MgSO}_{4}(\mathrm{pH} \mathrm{5.8)}$ for $2.5 \mathrm{~h}$ at $30^{\circ} \mathrm{C}$, and (ii) after transformation, the protoplasts were plated on a medium containing $0.8 \mathrm{M}$ sucrose, $10 \mathrm{mM}$ Tris, $1.5 \%$ Bacto agar (Difco Laboratories) and $100 \mu \mathrm{g}$ of $\mathrm{Hm}-\mathrm{B}$ (Calbiochem, San Diego, CA, U.S.A.) per ml with a $\mathrm{pH}$ adjusted to 7.4. To select the GFP-expressing, Hm-B-resistant cotransformants, the colonies were directly observed under a Zeiss Axioplan fluorescent microscope (Zeiss, Mannheim, Germany) with a 480-nm excitation/500-to-550 emission filter block.

Forty Hm-B-resistant transformants, corresponding to five transformants per $\mu \mathrm{g}$ of total DNA, were obtained and $60 \%$ appeared bright green fluorescent under the microscope. Variation of expression levels was detected among the transformants, and several Hm-B-resistant transformants were not fluorescent at all, presumably because of the absence of the $g f p$-carrying plasmid. After growth on all media used in this study, the level of $g f p$ expression in 19 single-spore isolated transformants was high in the vegetative and reproductive forms of the organism, including the mycelium, microconidia, macroconidia, and chlamydospores (Fig. 2A). Only vacuoles were not fluorescent and appeared as dark areas of various sizes within the cytoplasm. Four of the nineteen selected single-spore isolated transformants were tested for pathogenicity on tomato by artificial inoculations made in the gnotobiotic system (Simons et al. 1996). All were found as equally pathogenic as wild-type $F$. oxysporum f. sp. radicis-lycopersici, causing extensive crown and root damage or even death of 80 to $100 \%$ of the total number of plants within 7 days of growth in inoculated sand, as described below. One of these transformants, designated FCL14, was chosen for use in further experiments. After two successive transfers from PDA to tomato and back to PDA and nine transfers on PDA without the addition of Hm-B, the autofluorescence of FCL14 remained stable.

In order to prepare fungal inoculum for artificial inoculations on plants, F. oxysporum f. sp. radicis-lycopersici was grown in Armstrong medium (Singleton et al. 1992) on a shaker (130 to $160 \mathrm{rpm})$ at $28^{\circ} \mathrm{C}$ for 2 days. Subsequently, the culture was filtered through two layers of sterile Miracloth (Calbiochem Corp., La Jolla, CA, U.S.A.) and the filtrate containing the microconidia was centrifuged at $4,080 \times g$ for $15 \mathrm{~min}$. The supernatant was discarded, and the pellet was resuspended in $50 \mathrm{ml}$ of sterile distilled water and centrifuged again. In order to remove the Armstrong medium, this washing step was repeated four times. The microconidia were resuspended in sterile distilled water and used for inoculation of tomato seedlings. Tomato (Lycopersicon esculentum Mill.) cv. Carmello (Novartis Seeds, Enkhuizen, The Netherlands), which is susceptible to $F$. oxysporum f. sp. radicis-lycopersici, was used in all experiments. Sterilization of seeds was achieved as described by Simons and colleagues (1996). After sterilization, the seeds were placed on a plant nutrient solution (PNS) (Hoffland et al. 1989) solidified with $1.8 \%$ agar, incubated at $4^{\circ} \mathrm{C}$ for 2 days, and allowed to germinate at $28^{\circ} \mathrm{C}$ for 2 days. Artificial inoculations of $F$. oxysporum $\mathrm{f}$. sp. radicis-lycopersici on tomato were done in a gnotobiotic sand system (Simons et al. 1996). Sterile glass tubes were filled with sterilized quartz sand mixed to $10 \%$ (vol/wt) with PNS containing $5 \times 10^{2}$ spores per $\mathrm{ml}$, resulting in a final inoculum density of 50 spores per $g$ of sand. Germinated tomato seeds were planted in the contaminated sand.

Microscopic examinations started $24 \mathrm{~h}$ after inoculation and were continued daily for the first week and every 2 days for a second week using different plants for each time point. The plants were carefully taken out of the tubes and their roots were gently swirled a few times in tap water in order to wash away the sand particles. Whole roots were placed directly on glass slides in drops of water, covered with a cover glass, and examined. Observations were carried out in three different experiments.

Microscopic examinations were done with two fluorescent microscopes. One was a Leica DM IRBE (Leica, Mannheim, Germany) equipped with filter blocks with spectral properties matching those of GFP [470/20-nm excitation, 515 Long Pass emission $\left(\mathrm{I}_{3}\right)$ ] and the root's autofluorescence [538/22-nm excitation, 590 Long Pass emission $\left(\mathrm{N}_{2.1}\right)$ ], to which a TCS SP con-
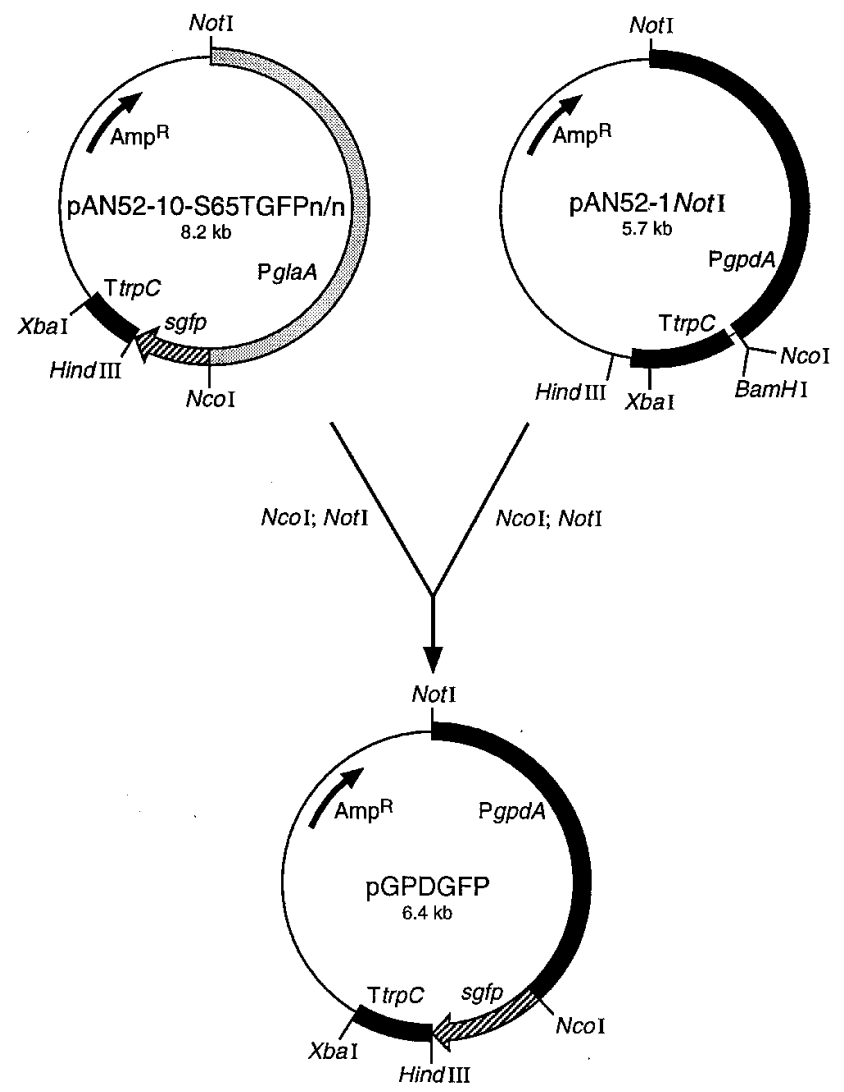

Fig. 1. Construction of a reporter plasmid to express $g f p$ in Fusarium oxysporum f. sp. radicis-lycopersici. Plasmid pGPDGFP was constructed to express $g f p$ under the control of the gpdA promoter in $F$. oxysporum $\mathrm{f}$. sp. radicis-lycopersici. Details on cloning are in text. 

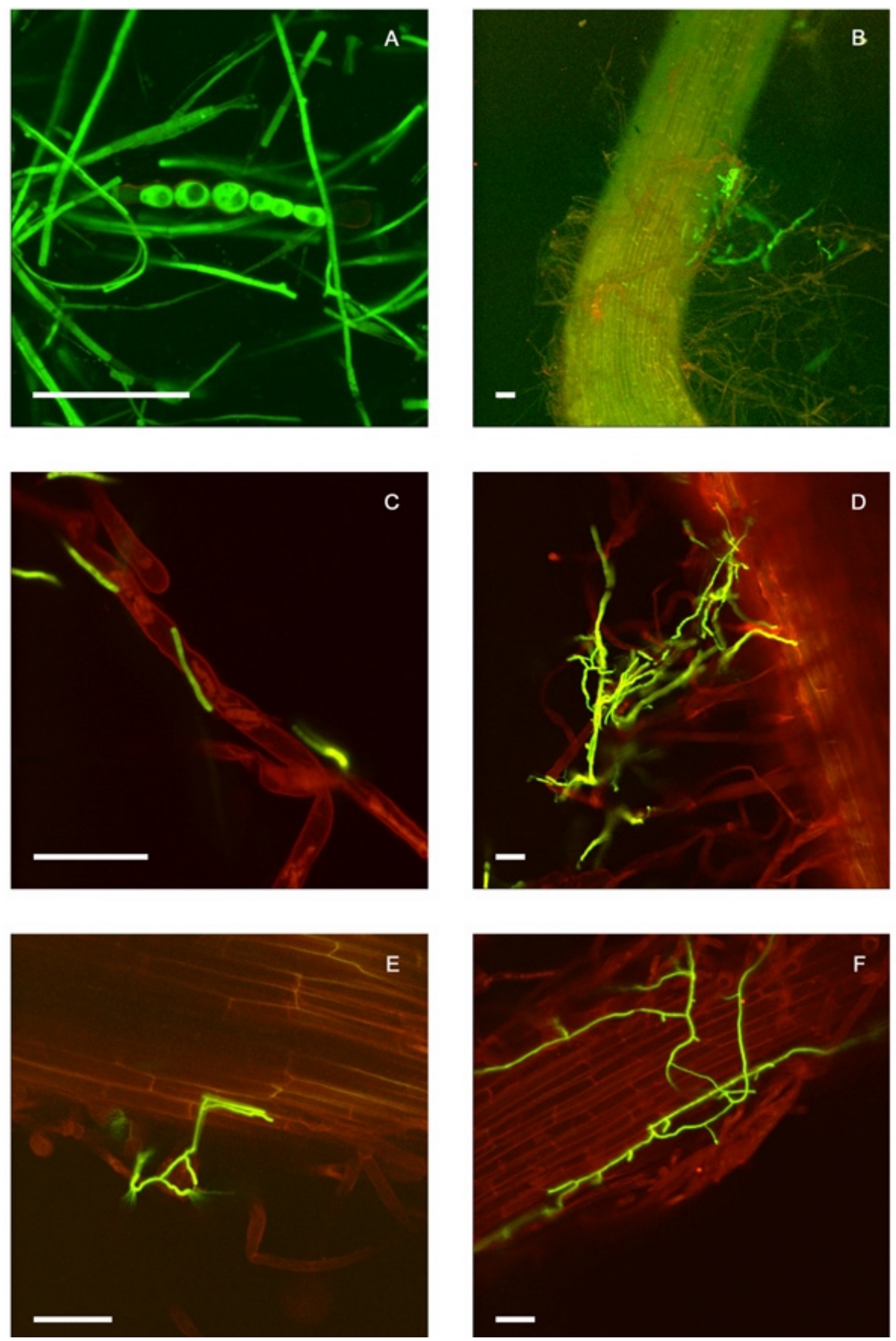

Fig. 2. Early stages of tomato root colonization by Fusarium oxysporum f. sp. radicis-lycopersici marked with gfp. Confocal scanning laser microscopy analyses of tomato roots grown after planting 2-day-old germinated sterile seedlings in sand containing spores of F. oxysporum f. sp. radicis-lycopersici. A, Uniform expression of $g f p$ in hyphae and chlamydospores of the transformed fungus grown on potato dextrose agar. B, Fungal hyphae in contact with tomato root hairs, 2 days after inoculation. C, Attachment of fungal hyphae to tomato root hairs, 2 days after inoculation. D, Intermingling of hyphae with root hairs at the crown region, 3 days after inoculation. E, Attachment of hyphae to the root surface and settling in the grooves between epidermal cells, 3 days after inoculation. $\mathbf{F}$, Colonization of the root surface by hyphae that are growing at the junctions of the epidermal cells, 3 days after inoculation. A to $\mathbf{F}, \mathrm{Scale}$ bar $=50 \mu \mathrm{m}$ 
focal scanhead was attached. The second was a Zeiss Axioplan equipped with filter blocks with spectral properties matching those of GFP (450-to-490-nm excitation, 520 Long Pass emission) and the root's autofluorescence (510-to-560-nm excitation, 590 Long Pass emission), coupled to an MRC 1024 SE Bio-Rad confocal system (Bio-Rad, Hercules, CA, U.S.A.).

Digital images were acquired by scanning with optimal settings for GFP: for the Leica TCS SP, excitation with the 488 Argon laser and detection of the emitted light between 520 to $540 \mathrm{~nm}$ (autofluorescence detection 600 to $670 \mathrm{~nm}$ ); for the Bio-Rad 1024, excitation with the 488 Argon/Krypton laser and detection of the emitted light at 522 DF32 (autofluorescence detection 585 Long Pass). The projections of the individual channels were merged in Photoshop 5.1 software (Adobe, San Jose, CA, U.S.A.) to facilitate visualization.

The $g f p$-labeled FCL14 was brightly fluorescent and readily visible on tomato roots even at a low (i.e., $\times 100$ ) magnification. No loss of fluorescence was observed during CLSM analyses. In a small number of cases, fluorescence was reduced by photobleaching, especially after repeated in depth scanning at the same site on the root. However, the cases of photobleaching were very few and not remarkable and did not affect the speed and quality of the microscopic study. The tomato root cell walls produce a natural red autofluorescent signal that improves the image by providing a clear view of the root and the position of the fungus in, on, or close to it. The sequence of events described here appeared in a similar way in all three replicates of the experiment.

One day after planting sterile germinated tomato seedlings in sand mixed with spores of $F$. oxysporum f. sp. radicis-lycopersici FCL14, all plants were apparently healthy and no mycelium was observed on or around the root. Two days after inoculation, all plants examined were healthy as observed macroscopically. However, the main root of most of them was surrounded by hyphae that were loosely interwoven with the root hairs and at random positions from the crown to the elongation zone with only the root tips remaining free of the presence of hyphae. In 2 out of 48 plants examined, loosely attached mycelium was observed in the elongation zone. Our observations show that the contact between the hyphae and the root was initiated predominantly at the root hairs (Fig. 2B) and that the hyphae were approaching the root surface after initial attachment to the root hairs (Fig. 2C).

After 3 days, none of the examined plants showed disease symptoms macroscopically, but microscopic examination showed that, on the majority of the plants, hyphae were densely interwoven with the root hairs (Fig. 2D). Close interaction between hyphae and root hairs was observed at random positions along the root, but for most of the plants, hyphae were predominantly present at the crown region. At several spots, attachment of hyphae to the root surface was observed (Fig. 2E), and after attachment, hyphae started to grow along the junctions of the epidermal cells (Fig. 2F). At this stage, no plant cell penetration was observed while the root tips still remained free of the presence of mycelium.

Four days after inoculation, approximately $60 \%$ of the examined plants still appeared healthy macroscopically, but growth of mycelium along the junctions of the epidermal plant cells had expanded to larger areas. Hyphae were characteristically outlining the epidermal cell borders, forming a fluorescent green pattern on the root surface (Fig. 3A and B). At the same time, on plants that macroscopically still appeared healthy, the first infection events were observed as judged by direct penetration of epidermal cells by hyphae that became swollen at the penetration site (Fig. 3C). No specific penetration structures such as appressoria were observed. Four days after inoculation, the remaining $40 \%$ of the plants exhibited brown discoloration at several spots along the root or at the crown, a typical symptom of foot and root rot. Most of the root surface was covered with more or less densely developed mycelium, and only the root tips still remained free of infection. At the spots where brown discoloration was visible, the hyphal network was denser and more complex (Fig. 3B), surrounding the root completely, especially at the crown region (Fig. 3D). At these sites, intercellular as well as intracellular growth of hyphae was observed in the epidermal cells and first layer of cortical cells. At the intracellular penetration points, hyphae were swollen and constricted, possibly because of pressure involved while growing through the narrow penetration pore that was formed (Fig. $3 \mathrm{E})$. Hyphae seemed to regain their original diameter after entering the cell (Fig. 3E).

Five days after planting, most of the plants had several brown spots along the main root, including the crown. The network of hyphae was tightly embracing most of the root surface and had now expanded to the tips, while the cells of the epidermal tissue had been fully colonized by hyphae (Fig. 3F). By that time, colonization of lateral roots had also taken place. This did not necessarily start at the sites of emergence but seemed to happen at random positions (Fig. 4A and B). Hyphae were also observed growing inside root hairs (Fig. 4C).

Six days after inoculation, approximately 20 to $30 \%$ of the plants showed wilting symptoms and loss of firmness. Another approximately 60 to $70 \%$ of the plants exhibited extensive damage of their roots, while a small number of plants (approximately $10 \%$ ) showed symptoms of earlier stages of the disease. At heavily infected sites, the root tissue was not visible anymore, since all the tissues were fully overgrown and infected by the fungus (Fig. 4D). By that time, hyphae had colonized the central cylinder and were growing in xylem vessels (Fig. 4E).

Over the next 4 days, the disease progressed further on all plants, eventually resulting in senescence and decay. After fully colonizing the root system and destroying it completely, the fungus started to sporulate on the decayed roots, forming macroconidia (Fig. 4F), and to colonize the collapsed cotyledons.

Transformation of $g f p$ has been reported for 18 fungal/ fungallike species belonging to 12 genera, namely Ustilago, Aureobasidium, Colletotrichum, Mycosphaerella, Magnaporthe, Cochliobolus, Trichoderma, Podospora, Sclerotinia, Schizophyllum, Aspergillus, and Phytophthora (Lorang et al. 2001). In this paper, transformation and expression of the $g f p$ gene in the phytopathogenic fungus $F$. oxysporum f. sp. radicislycopersici are described for the first time. The transformation method was successful, as indicated by the relatively large number of fluorescent transformants obtained. Fluorescent transformants showed stable expression after numerous generations on nutrient media and on the plant. In addition, the fluorescent signal was strong and homogeneous in both vegetative and reproductive forms of the fungus (Figs. 2A and 4F). The variability of expression levels observed among transformants can be explained by the possible integration of the plasmid at different chromosomal locations. The transformants tested, randomly selected among those giving the highest GFP expression, showed a phenotype indistinguishable from that of the wild type with respect to growth rate, colony morphology, and pathogenicity on tomato. The fungus could easily be observed around, on, and within the tomato root (Figs. 2 to 4). Using CSLM, observations could start without any special preparation of the material, such as the use of chemicals for fixing or staining, which hastened work during microscopic analyses. GFP's fluorescence was strong and stable for several hours during observations, and no problems due to photobleaching were encountered. Autofluorescence of the tomato root cell walls did not interfere with the fluorescence of GFP. In fact, it facilitated the observations since it clearly showed the presence 

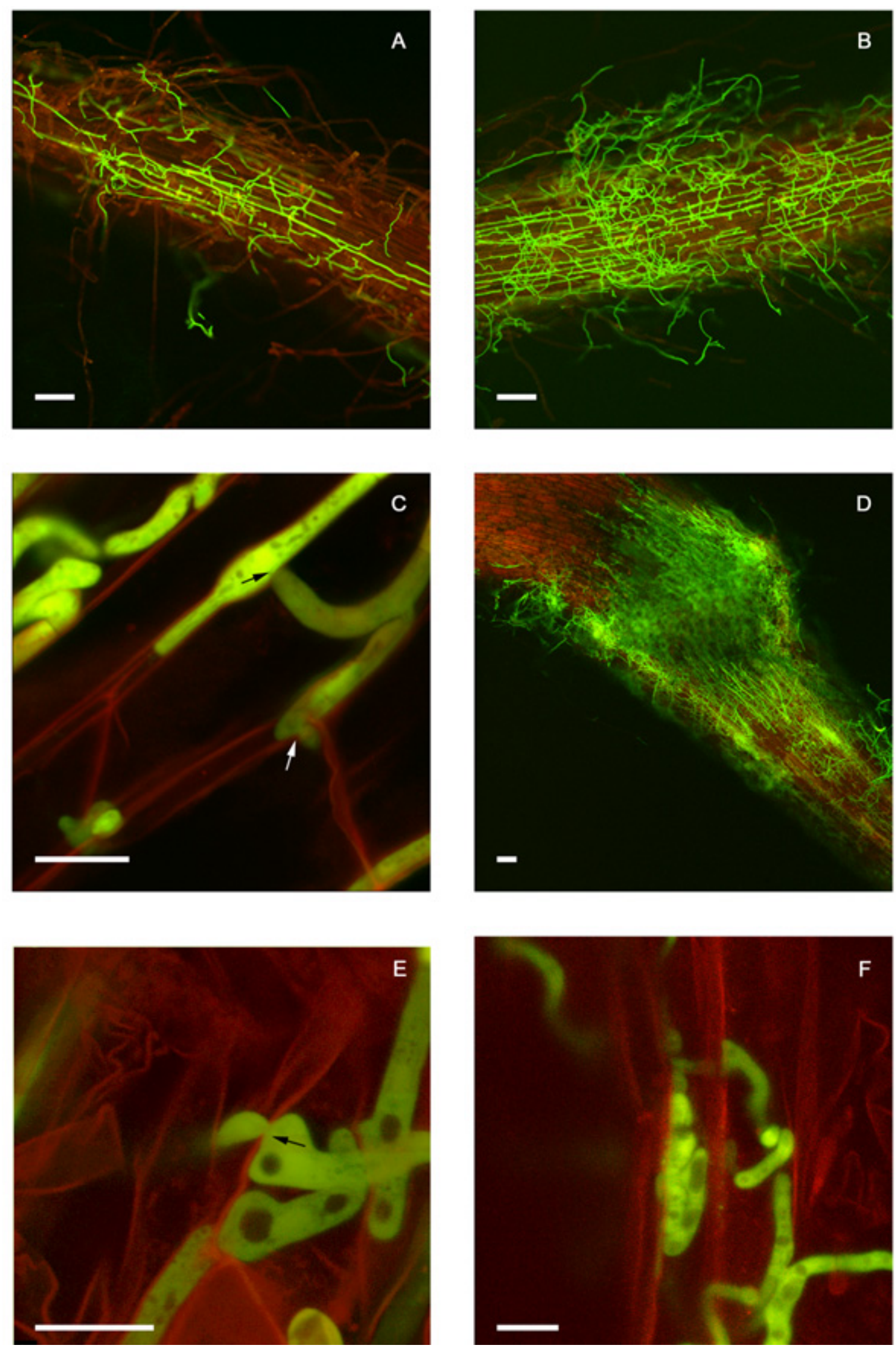

Fig. 3. Advanced stages of tomato root colonization and several stages of infection by Fusarium oxysporum f. sp. radicis-lycopersici marked with gfp. Confocal scanning laser microscopy analyses of tomato roots grown after planting 2-day-old germinated sterile seedlings in sand containing spores of $F$. oxysporum f. sp. radicis-lycopersici. A and B, Two subsequent steps in root surface colonization by hyphae growing at the junctions of the epidermal cells and forming an expanding network, 4 days after inoculation. C, A hypha growing at the junction of two epidermal cells, penetrating one of them (black arrow) and advancing to an adjacent cell (white arrow), 4 days after inoculation. D, Fully colonized crown of a seedling that exhibits crown rot symptoms, 4 days after inoculation. E, Intracellular growth of hyphae in root cells with a clearly visible penetration pore (indicated by arrow), 4 days after inoculation. F, Hyphae growing in epidermal root cells, 5 days after inoculation. A, B, and D, Scale bar $=50 \mu \mathrm{m}$ and $\mathbf{C}, \mathbf{E}$, and F, scale bar $=10 \mu \mathrm{m}$. 

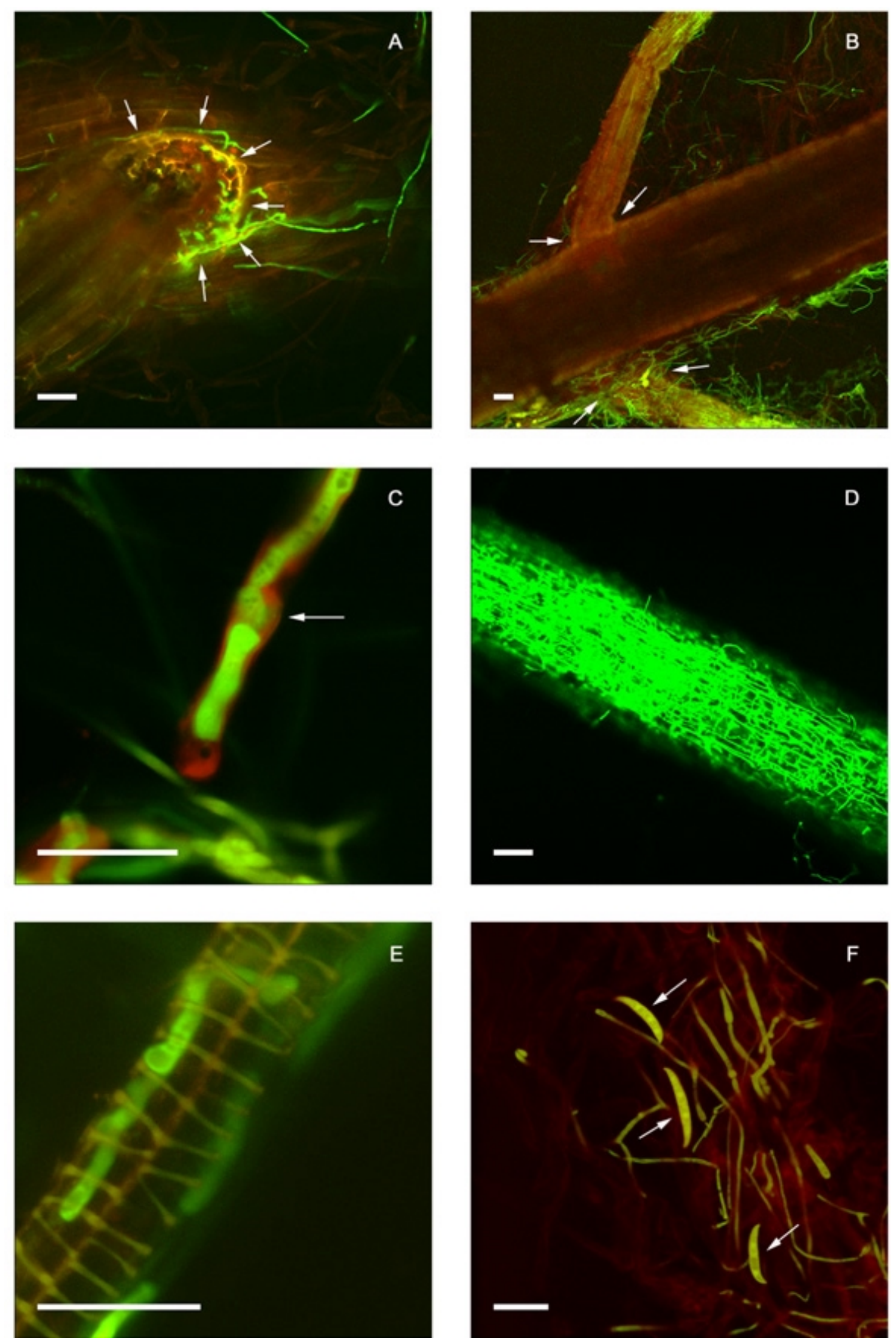

Fig. 4. Late stages of tomato root colonization and infection by Fusarium oxysporum f. sp. radicis-lycopersici marked with gfp. Confocal scanning laser microscopy analyses of tomato roots grown after planting 2-day-old germinated sterile seedlings in sand containing spores of $F$. oxysporum f. sp. radicislycopersici. Colonization of a lateral root $\mathbf{A}$, at the emergence site or $\mathbf{B}$, at random sites, 5 days after inoculation (the site of emergence of lateral roots is indicated by arrows). $\mathbf{C}$, A hypha growing in a root hair (indicated by arrow), 5 days after inoculation. D, Fully colonized root of a seedling that exhibits extensive root rot, 6 days after inoculation. E, Hyphae growing in a xylem vessel of a fully colonized root that exhibits rot symptoms, 6 days after inoculation. F, Macroconidia (indicated by arrows) of the fungus formed on a fully colonized and decayed root, 10 days after inoculation. A to D and F, Scale bar $=50 \mu \mathrm{m}$ and $\mathbf{E}$, scale bar $=10 \mu \mathrm{m}$. 
of the individual root cells (Figs. 2 to 4). Additional staining, e.g., with propidium iodide in order to visualize the root cells, was therefore not necessary.

The results of this work demonstrate that GFP is an excellent marker for studying $F$. oxysporum f. sp. radicis-lycopersicitomato in vivo interactions in the rhizosphere using CSLM. At the same time, this technique has several advantages compared with others, e.g., scanning electron microscopy (SEM) or GUS staining: (i) faster processing of the plant material and avoidance of the use of harmful chemicals; (ii) less influence by potential artifacts due to the absence of chemicals; (iii) preservation of decayed tissue and fragile fungal structures, e.g., conidia, which allowed monitoring the complete digestion of the root (Fig. 4D) and the sporulation of the fungus on the decayed tissue (Fig. 4F), since the fragile spores and tissues were not subjected to any harmful staining agent; and (iv) maintenance of the living status of organisms, an essential detail that allows time-lapse observations in vivo. This last advantage will open the way for a much more detailed study of $F$. oxysporum f. sp. radicis-lycopersici-tomato in vivo interactions in the rhizosphere using video microscopy.

Several aspects of this study on the tomato root colonization and infection processes by $g f p$-labeled $F$. oxysporum f. sp. radicis-lycopersici confirmed earlier reports of studies carried out using SEM (Brammall and Higgins 1988; Charest et al. 1984). However, besides the detailed observations at the cellular level (Figs. 3C, E, and F, and 4C and E), the use of GFP provided new information on the colonization and infection processes not described so far. First, it provided a clear overview in time of the tomato root system colonized by the fungus (Figs. 2B to F; 3A, B, and D; and 4A, B, D, and F). Second, details of the very early colonization steps, involving the recognition process between the two organisms in the beginning of the disease development, were visualized. It was demonstrated, for the first time, that the fungus is initially approaching the root via the root hairs (Fig. 2B to D). Third, it showed that preferable colonization sites on the root surface are the junctions along the epidermal cells, where the fungus starts to attach its growing hyphae soon after approach via the root hairs (Fig. 2E). While colonizing the root surface, the fungus forms a network of hyphae that grow and fill all the junctions of the epidermal cells (Figs. $2 \mathrm{~F}$ and $3 \mathrm{~A}$ and $\mathrm{B}$ ). The development of this hyphal network is faster and richer at the crown region (Fig. 3D). Finally, it showed that primary infection sites are junctions of epidermal root cells at random positions on the root (Fig. 3C) but not the root tip or sites of emergence of secondary roots. Root tips are infected at a later stage and secondary roots are infected either at the emergence site or at random positions (Fig. 4A and B). Also, wounding of the root tissue is not necessary for initiation of infection, since the fungus is able to penetrate the cells directly (Fig. 3C).

However, some limitations regarding the localization of the initial contact of the fungus and the plant should be considered. Mycelium that is close but still not attached to the roots could possibly be removed during the (gentle) sample preparation. It is possible that mycelium originating from spores in the sand is growing toward the elongation zone at the same time that it is growing toward the root hair zone of the root. However, mycelium close or loosely attached to the root surface in the elongation zone was seldom observed. Based on these observations, colonization and infection are most likely to start in the root hair zone rather than the elongation zone. It seems like hyphae have a stronger chemotactic response toward, or can better entangle, the root hairs. Our observations show that the disease progresses faster at the root hair zone, even if the elongation zone might be approached at the same time. Several locations along the root hair zone, and especially the crown, are the first to be infected as observed microscopically, and subsequently macroscopically, by the presence of initial disease symptoms, like brown discoloration. Root tips are infected by mycelium that has grown on the root rather than colonized and infected directly from the sand.

Differences in the time of initiation of infection or disease progress between this and previously reported works (Brammall and Higgins 1988; Charest et al. 1984) are most likely because of the experimental design. Deposition of the inoculum directly on the root (Brammall and Higgins 1988; Charest et al. 1984) allows infection to start faster (as soon as 1 day after inoculation), since the fungal propagules are in direct contact with the root surface. Mixing the inoculum in the planting material (sand or soil), an approach used in this work because it better resembles the conditions of the infested soils in nature, suggests that the fungal spores need some time to react to the presence of the growing root, to germinate, and to reach the root, causing a delay of the infection process.

This work also has shown differences and similarities regarding the primary infection strategy of $F$. oxysporum $\mathrm{f}$. sp. radicis-lycopersici in comparison to other formae speciales of $F$. oxysporum. It was demonstrated that the tomato root tip does not seem to be of great importance as a primary infection site for $F$. oxysporum f. sp. radicis-lycopersici. This is in contrast to the cases of other wilt-causing formae speciales of $F$. oxysporum, in which the root tip is of primary significance as a site for disease initiation (Farquhar and Peterson 1989; Smith and Peterson 1983). In the case of the tomato wilt pathogen $F$. oxysporum $\mathrm{f}$. sp. lycopersici, the root tip is included among other sites of initial infection (Olivain and Alabouvette 1999). The intense colonization of the root hair zone by $F$. oxysporum f. sp. radicis-lycopersici is a characteristic in common with the infection by $F$. oxysporum f. sp. lycopersici (Olivain and Alabouvette 1999).

Knowledge of the colonization strategy of $F$. oxysporum $\mathrm{f}$. sp. radicis-lycopersici on tomato roots is essential for studies on the efficiency of colonization strategies by antagonistic microorganisms that are able to suppress foot and root rot disease, such as Pseudomonas biocontrol bacteria (Chin-AWoeng et al. 1997; Dekkers et al. 2000). Dual labeling of the two organisms with different autofluorescent proteins will allow the detailed analysis of direct interactions between biocontrol agents and F. oxysporum $\mathrm{f}$. sp. radicis-lycopersici in the tomato rhizosphere and could contribute to the development of more efficient ways to control the disease.

\section{ACKNOWLEDGMENTS}

This research was financially supported by a Marie Curie Fellowship of the European Community program Training and Mobility of Researchers under contract ERBFMBICT982930. The authors thank J. Mes and H. Teunissen from the Institute of Molecular Cell Biology, BioCentrum Amsterdam, for their useful suggestions on modification of the transformation procedure and Novartis Seeds, Enkhuizen, The Netherlands, for providing certified 'Carmello' seeds.

\section{LITERATURE CITED}

Brammall, R. A., and Higgins, V. J. 1988. A histological comparison of fungal colonization in tomato seedlings susceptible or resistant to Fusarium crown and root rot disease. Can. J. Bot. 66:915-925.

Brayford, D. 1996. Fusarium oxysporum f. sp. radicis-lycopersici, IMI descriptions of fungi and bacteria no. 1270. Mycopathologia 133:61-63.

Chalfie, M., and Kain, S. 1998. GFP Green Fluorescent Protein Properties, Applications and Protocols. Wiley-Liss, Inc., New York.

Charest, P. M., Quellette, G. B., and Pauze, F. J. 1984. Cytological observations of early infection process by Fusarium oxysporum f. sp. radicis-lycopersici in tomato plants. Can. J. Bot. 62:1232-1244.

Chin-A-Woeng, T. F. C., de Priester, W., van der Bij, A. J., and Lugtenberg, B. J. J. 1997. Description of the colonization of a gnotobi- 
otic tomato rhizosphere by Pseudomonas fluorescens biocontrol strain WCS365, using scanning electron microscopy. Mol. Plant-Microbe Interact. 10:79-86.

Chin-A-Woeng, T. F. C., Bloemberg, G. V., van der Bij, A. J., van den Drift, K. M. G. M., Schripsema, J., Kroon, B., Scheffer, R. J., Keel, C., Bakker, P. A. H. M., Tichy, H.-V., de Bruijn, F. J., Thomas-Oates, J. E., and Lugtenberg, B. J. J. 1998. Biocontrol by phenazine-1-carboxamide-producing Pseudomonas chlororaphis PCL1391 of tomato root rot caused by Fusarium oxysporum f. sp. radicis-lycopersici. Mol. Plant-Microbe Interact. 11:1069-1077.

Chiu, W. L., Niwa, Y., Zeng, W., Hirano, T., Kobayashi, H., and Sheen, J. 1996. Engineered GFP as a vital reporter in plants. Curr. Biol. 6:325330.

Dekkers, L. C., Mulders, I. H. M., Phoelich, C. C., Chin-A-Woeng, T. F. C., Wijfjes, A. H. M., and Lugtenberg, B. J. J. 2000. The sss colonization gene of the tomato-Fusarium oxysporum $\mathrm{f}$. sp. radicis-lycopersici biocontrol strain Pseudomonas fluorescens WCS365 can improve root colonization of other wild-type Pseudomonas spp. bacteria. Mol. PlantMicrobe Interact. 13:1177-1183.

Farquhar, M. L., and Peterson, R. L. 1989. Pathogenesis of Fusarium root rots of Pinus resinosa in test tubes. Can. J. Plant Pathol. 11:221-228.

Hoffland, E., Findenegg, G. R., and Nelemans, J. A. 1989. Solubilization of rock phosphate by rape. Plant Soil 113:161-165.

Jarvis, W. R. 1988. Fusarium crown and root rot of tomatoes. Phytoprotection 69:49-64.

Kistler, H. C., and Benny, U. K. 1988. Genetic transformation of the fungal wilt pathogen, Fusarium oxysporum. Curr. Genet. 13:145-149.

Lorang, J. M., Tuori, R. P., Martinez, J. P., Sawyer, T. L., Redman, R. S. Rollins, J. A., Wolpert, T. J., Johnson, K. B., Rodriguez, R. J., Dickman, M. B., and Ciuffetti, L. M. 2001. Green fluorescent protein is lighting up fungal biology. Appl. Environ. Microbiol. 67:1987-1994.

Lugtenberg, B. J. J., Dekkers, L., and Bloemberg, G. V. 2001. Molecular determinants of rhizosphere colonization by bacteria. Annu. Rev. Phytopathol. 39:461-490

Maniatis, T. A., Fritsch, E. F., and Sambrook, J. 1982. Molecular Cloning: A Laboratory Manual. Cold Spring Harbor Laboratory, Cold Spring Harbor, NY, U.S.A.
Mes, J. J., Wit, R., Testerink, C. S., de Groot, F., Haring, M. A., and Cornelissen, B. J. C. 1999. Loss of avirulence and reduced pathogenicity of a gamma-irradiated mutant of Fusarium oxysporum f. sp. lycopersici. Phytopathology 89:1131-1137.

Mullaney, E. J., Hamer, J. E., Roberti, K. A., Yelton, M. M., and Timberlake, E. 1985. Primary structure of the $\operatorname{trp} C$ gene from Aspergillus nidulans. Mol. Gen. Genet. 199:37-45.

Olivain, C., and Alabouvette, C. 1999. Process of tomato root colonization by a pathogenic strain of Fusarium oxysporum f. sp. lycopersici in comparison with a non-pathogenic strain. New Phytol. 141:495-510.

Punt, P. J., Oliver, R. P., Dingemanse, M. A., Powels, P. H., and Van den Hondel, C. A. M. J. J. 1987. Transformation of Aspergillus based on the hygromycin-B marker from Escherichia coli. Gene 56:117-124.

Punt, P. J., Dingemanse, M. A., Jacobs-Meijsing, B. J. M., Pouwels, P. H., and Van den Hondel, C. A. M. J. J. 1988. Isolation and characterization of the glyceraldehyde-3-phosphate dehydrogenase gene of Aspergillus nidulans. Gene 69:49-57.

Siedenberg, D., Mestric, S., Ganzlin, M., Schmidt, M., Punt, P. J., Van den Hondel, C. A. M. J. J., and Rinas, U. 1999. GlaA promoter controlled production of a mutant green fluorescent protein (S65T) by recombinant Aspergillus niger during growth on defined medium in batch and fed-batch cultures. Biotechnol. Prog. 15:43-50.

Simons, M., van der Bij, A. J., Brand, I., de Weger, L. A., Wijffelman, C. A., and Lugtenberg, B. J. J. 1996. Gnotobiotic system for studying rhizosphere colonization by plant growth-promoting Pseudomonas bacteria. Mol. Plant-Microbe Interact. 9:600-607.

Singleton, L. L., Mihail, J. D., and Rush, C. M., eds. 1992. Methods for Research on Soilborne Phytopathogenic Fungi. American Phytopathological Society, St. Paul, MN, U.S.A.

Smith, A. K., and Peterson, R. L. 1983. Examination of primary roots of asparagus infected by Fusarium. Scanning Electron Microsc. 3:14751480 .

Tsien, R. Y. 1998. The green fluorescent protein. Annu. Rev. Biochem. 67:509-544.

Van den Hondel, C. A. M. J. J., Punt, P. J., and van Gorgom, R. F. M. 1991. Heterologous gene expression in filamentous fungi. Pages 398 428 in: More Gene Manipulations in Fungi. Academic Press, New York. 